\title{
EDITORIAL
}

\section{PLOTTING THE TRAJECTORIES OF TOMORROW'S MAGIC BULLETS}

\author{
This month's focus issue highlights the great potential of pharmacogenetics to deliver more \\ tailored healthcare. Preparation for this revolution should include honest assessment of the \\ cost of future medicines.
}

This year marks the one hundred and fiftieth anniversary of the birth of Paul Ehrlich — Nobel laureate, inventor of salvarsan as a treatment for syphilis and coiner of the term 'magic bullet'. These words, as originally formulated, referred to chemical substances that can be used to destroy pathogenic organisms by virtue of their particular affinity for such pathogens. Nowadays, 'magic bullets' are more frequently envisaged as being side-effect-free drugs specifically targeted to particular individuals with particular diseases. This evolution in terminology is mirrored in this issue, with the inclusion of both a sesquicentennial anniversary 'Timeline' on Paul Ehrlich's most significant scientific contributions, and a collection of articles on the promise and current status of pharmacogenetics, a discipline which seeks to refine drug therapy through the drug-specific segmentation of patient populations and the elimination of adverse events.

The focus on pharmacogenetics - run jointly with Nature Reviews Genetics, which is publishing several complementary articles - brings together the views of leading academics, regulators and industry researchers. Although the anticipated 'revolution' in healthcare that pharmacogenetics promises has not yet occurred, the community is clearly getting better at defining the questions that need to be addressed to achieve the full potential benefits of pharmacogenetics. For instance, although there is still not a clear picture of the statistical power needed to define confidence limits for pharmacogenetic data sets, or of the receptivity of regulators worldwide for such data, we can at least have confidence that these topics are being very actively investigated.

There is, however, one major question surrounding medicines that we tend to ignore, and that is the issue of cost. The key concern is not the cost of current drugs, although this is clearly a topic of great importance, but the cost of future drugs, and how we will come to terms with the fact that many of the new healthcare products anticipated to emerge in coming decades will be targeted not just to pharmacogenetically segmented patient populations, but to economically segmented populations too.

The economic restriction of healthcare provision is already a burning issue, with billions of people around the world having almost no access to drugs at all, but within the major markets the prevailing view is still that we have the right, or at least should have the right, to the best available drugs to treat our diseases. Current negotiations to determine the drug class definitions for Medicare's formulary in the United States, for instance, are centred around the debate on whether the system will leave room for pharmacy-benefit managers to substitute generic versions of older, less effective therapeutics for newer, more expensive medicines. This practice of restricting access to the latest therapies strikes many as inherently unfair. But as the gap between affordable, older medicines and newer therapies widens, there must surely come a time when even those in the most affluent markets will have to come to terms with the fact that these new medicines will be luxury items.

That time is not with us yet. The public debate has yet to tackle the issue of how society will handle future drug pricing, and is instead still focused on the perceived unfairness of certain new drugs' premium pricing, and calls for drug prices to be generally lowered. Such lowering would create short-term relief, but the costs of discovering and developing drugs will only increase as time goes on, and the medicines of the future will be more and more expensive. People demand better drugs, and those who can afford the best drugs will be willing to pay for them. But those who can pay will be an ever-diminishing proportion of the population, and the rest will have to make do with what they can afford. Recognizing and openly discussing this harsh fact is a prerequisite for entering into sensible policy discussion about what sort of medicines we truly want the pharmaceutical industry to provide us with in future. 